\title{
Sobrevivência e crescimento inicial de Ocotea pulchella (Lauraceae) em uma floresta de restinga da Ilha do Cardoso, SP
} Survival and initial growth of Ocotea pulchella (Lauraceae) in a restinga forest at Ilha do Cardoso, SP

\author{
Luciana Andréa Pires ${ }^{1,5}$, Victor José Mendes Cardoso ${ }^{2}$, Ricardo Ribeiro Rodrigues ${ }^{3} \&$ Carlos Alfredo Joly ${ }^{4}$
}

\begin{abstract}
Resumo
Neste trabalho, avaliou-se os efeitos de fatores ambientais na sobrevivência e crescimento inicial de Ocotea pulchella (Nees) Mez numa floresta de restinga. Os ensaios consistiram do plantio de mudas classificadas em dois grupos de idade (plântulas e juvenis) em diferentes áreas selecionadas quanto à cobertura vegetal (clareiras ou sub-bosque) e umidade do solo (secos e úmidos). As plantas de ambas as idades apresentaram a maior taxa de mortalidade no sub-bosque úmido, onde se observou os valores mais baixos de luz fotossinteticamente ativa e fertilidade do solo. A sobrevivência de plântulas correlacionou-se positivamente ao crescimento, que por sua vez, foi favorecido em clareiras, sugerindo uma dependência da espécie em relação a ambientes mais iluminados, embora essa possa ocupar o sub-bosque em fisionomias mais abertas como as restingas. Em juvenis, a sobrevivência foi relacionada principalmente aos fatores edáficos; o crescimento em altura foi muito baixo, não sendo constatado incremento significativo de área foliar nem de matéria seca em nenhum dos ambientes, o que pode estar relacionado ao caráter oligotrófico do solo. Os juvenis em sub-bosque de solo úmido apresentaram ao final do experimento os menores valores de altura, área foliar, razão de área foliar, razão de massa foliar e taxa de assimilação líquida, sugerindo que baixas irradiâncias associadas a solos saturados e quimicamente pobres podem restringir o recrutamento da espécie no local estudado. Todavia, o comportamento de certa forma generalista para as condições de luz e umidade do solo, indica uma alta capacidade de $O$. pulchella em colonizar distintos micro-ambientes da restinga, onde se observa uma grande variação espaço-temporal de fatores ambientais.
\end{abstract}

Palavras-chave: plântula; regeneração; microambiente; Ilha do Cardoso; restinga.

\begin{abstract}
The aiming of this work was to evaluate the effects of environmental factors on both the survival and initial growth of Ocotea pulchella (Nees) Mez at a restinga forest. The tests consisted of transplanting seedlings classified into two age groups (seedlings and juveniles) in different areas selected as for plant covering (gaps or understorey) and soil moisture (dry or wet). Both seedlings and juveniles presented the highest mortality rate when they were planted in the 'wet understorey', in which the lowest soil fertility and irradiance levels were recorded. Seedling survival correlated positively with growth, which was promoted in gaps, suggesting a dependence of the species on more lighted environments, although $O$. pulchella can occupy the understorey in more open physiognomies such as restinga forest. The juveniles survival correlated mainly with edaphic factors; the growth in height was very low and no significant increase in leaf area and dry weight was observed in any of the environments, what can be related to the oligotrophic characteristics of the soil. Juveniles planted in 'wet understorey' presented the lowest height, leaf area, leaf area ratio, leaf mass ratio and net assimilation rate values, suggesting that the species recruitment in the restinga can be reduced in environments with low irradiances and waterlogged soils. However, the somehow generalist character of the species in response to irradiance levels and soil moisture may suggest a high ability of $O$. pulchella in colonizing distinct microenvironments at restinga, in which environmental factors may fluctuate greatly over time and space.
\end{abstract}

Key words: seedling; regeneration; micro-environment; Ilha do Cardoso; sandy coastal plane.

\footnotetext{
${ }^{1}$ Universidade Norte do Paraná, Centro de Ensino a Distância, R. Tiête 1208, 86025-230, Londrina, PR.

${ }^{2}$ Universidade Estadual Paulista, IB, Depto. Biologia Vegetal, Av. 24a, 1515, 13506-900, Rio Claro, SP.

${ }^{3}$ Universidade de São Paulo, Escola Superior de Agricultura Luiz de Queiróz, Depto. Ciências Biológicas, C.P. 9, 13418-900, Piracicaba, SP.

${ }^{4}$ Universidade Estadual de Campinas, IB, Depto. Biologia Vegetal, C.P. 6109, 13083-970, Campinas, SP.

${ }^{5}$ Autor para correspondência: luapires@hotmail.com
} 


\section{Introdução}

O estabelecimento de plântulas arbóreas, por ser altamente vulnerável, é reconhecido como uma das fases mais críticas para a regeneração das florestas tropicais (Augspurger 1984; Clark \& Clark 1992; Muller-Landau et al. 2002). A principal abordagem das pesquisas relacionadas a este processo é o comportamento das espécies em relação aos níveis de luz, umidade e nutrientes do solo, os quais afetam, diferencialmente, a germinação, sobrevivência, o crescimento e morfologia das plantas (Bazzaz \& Picket 1980; Pompa \& Bongers 1988; Clark 1990; Denslow 1996; Poorter 1999). A adequação morfológica das plântulas às condições ambientais está associada ao sucesso reprodutivo da espécie (Garwood 1996), sendo relatado para muitas delas, alterações anatômicas e fisiológicas nas folhas e/ou na proporção de tecidos fotossintetizantes em relação aos não fotossintetizantes das plântulas, quando submetidas a diferentes situações de luz e umidade do solo (Pompa \& Bongers 1988; Souza \& Valio 2003; Kozlowski et al. 1991).

A vegetação de restinga ocorre sobre a planície arenosa originada no quaternário pela deposição marinha, sendo bastante heterogênea, florística e estruturalmente, em decorrência, entre outros, das características edáficas. A condição de drenagem do solo depende principalmente da microtopografia do terreno e do nível do lençol freático que varia conforme as condições climáticas, podendo gerar um gradiente espaço-temporal de locais secos até inundáveis em áreas contíguas (Araujo 1992; Sugiyama 1998; Silva \& Britez 2005; Magnago et al. 2010). Tais fatores, além da dinâmica de clareiras, são considerados componentes fundamentais para a renovação florestal e devem influenciar o recrutamento das plantas, sendo esperado que as espécies possuam características morfofisiológicas que as permita colonizar esses gradientes.

A flora de restinga possui pouco endemismo, sendo composta por espécies originadas de formações vegetacionais mais antigas (Araújo \& Henriques 1984), e que foram capazes de se adaptar às condições ambientais adversas, tais como a alta radiação, oligotrofia, salinidade, inundações e seca (Lacerda et al. 1993; Franco et al. 1996). Mesmo com a alta variabilidade ambiental, algumas espécies aparentemente tem uma distribuição ampla dentro da paisagem. Dentre estas figura Ocotea pulchella (Nees) Mez, árvore zoocórica que ocorre nas Regiões Centro-Oeste, Sudeste e Sul do Brasil, sendo frequente e em restingas (Carvalho 2006;
Sugiyama 1998; Silva \& Britez 2005), cerrado (Durigan et al. 2002; Pinheiro \& Monteiro 2006), floresta estacional (Pinheiro \& Monteiro 2009), matas de galeria (Rodrigues \& Naves 2004, Pinto et al. 2005), floresta ombrófila mista (Formento et al. 2004) e, menos comumente, em floresta ombrófila densa (Brotto et al. 2009). No estado São Paulo está na lista de espécies indicadas para utilização em reflorestamentos de todos estes ecossistemas (São Paulo 2003). Como reflexo da ocorrência em diferentes condições de luz, tais como áreas antropizadas, bordas e interior de florestas (Salimon \& Negrelle 2001; Carvalho 2006), O. pulchella já foi classificada como secundária inicial em floresta estacional (Pinheiro \& Monteiro 2009), e pioneira antrópica, secundária inicial e tardia, em formações vegetais da planície costeira (Salimon \& Negrelle 2001; Carrasco 2003; Sugiyama 2003). Estudos em restingas (Carrasco 2003, Sugyama 2003; Silva \& Britez 2005) mostram que O. pulchella está presente desde formações arbustivas até florestais, apresentando menor densidade em áreas de maior saturação hidrica do solo e menor porte em solos mais secos.

Este trabalho teve como objetivo avaliar, em diferentes microambientes discriminados quanto às condições de luz ("clareira" e "sub-bosque") e umidade do solo ("secos" e "úmidos"), a sobrevivência e crescimento de plântulas e juvenis de Ocotea pulchella em uma floresta de restinga alta no Parque Estadual da Ilha do Cardoso/SP, onde a espécie é abundante (Lima et al. 2011), sendo esperado que a alta saturação hídrica restrinja o recrutamento da espécie. Pretende-se, desta forma, contribuir com o entendimento da dinâmica da regeneração neste ecossistema altamente ameaçado pela ação antrópica.

\section{Material e Métodos}

Área de estudo

Os ensaios de campo foram realizados numa área de 10,24 ha, onde foram demarcadas parcelas de $20 \times 20 \mathrm{~m}\left(25^{\circ} 04^{\prime} 41^{\prime \prime} \mathrm{Se}\right.$ e $7^{\circ} 55^{\prime}$ '53"'W) em floresta

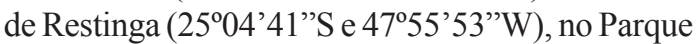
Estadual da Ilha do Cardoso (PEIC), Cananéia - SP.

Segundo a classificação de Köppen a Ilha possui clima tipo Af, sendo que as médias de temperaturas, mínimas e máximas, giram em torno de $19^{\circ} \mathrm{C} \mathrm{e} 27^{\circ} \mathrm{C}$, respectivamente, e precipitação média anual, entre 1.800 e $2.000 \mathrm{~mm}$ (Melo \& Mantovani 1994). Na área de estudo, os solos foram classificados como hidromórficos, arenosos, muito ácidos, com 
teores variáveis de matéria orgânica, distribuindo-se conforme a idade e a microtopografia, que reflete a influência do lençol freático (Gomes et al. 2007).

Os microambientes estudados neste trabalho foram selecionados quanto à abertura do dossel e umidade do solo sendo agrupados como: sub-bosque seco (SS); sub-bosque úmido (SU); clareira seca (CS) e; clareira úmida (CU). Foram consideradas "clareiras" áreas com aberturas no dossel maiores do que $4 \mathrm{~m}^{2}$; os níveis de umidade foram distinguidos pela elevação do terreno e características do solo (cor, textura, evidências de alagamento) e da vegetação, conforme descrição dos tipos florestais em diferentes gradações de umidade do solo por Silva \& Britez (2005). Os ambientes de solo "seco", com textura mais arenosa e sem evidência de alagamento, ficaram localizados em locais mais elevados, onde a espessura do horizonte eluvial e a profundidade do lençol freático são maiores do que nas zonas mais baixas, onde ficaram situados os pontos de solo úmido, com saturação hídrica permanente. De acordo com o mapa de solos da parcela (Gomes et al. 2007), pode ser observado que os pontos de amostragem ficaram situados em espodossolo hidromórfico, embora os do sub-bosque úmido, localizados em condição de um pequeno abaciamento do terreno, apresentaram associação com organossolos, assim classificados devido ao elevado acúmulo de matéria orgânica na superfície.

\section{Material vegetal}

Foram utilizadas plântulas de Ocotea pulchella com aproximadamente três meses após a germinação, obtidas de sementes coletadas no Parque Estadual da Ilha do Cardoso e germinadas em bandejas plásticas preenchidas com composto à base de casca de Pinus moída e vermiculita, mantidas sob luz natural direta em Casa de Vegetação na UNESP/ Rio Claro. Também foram utilizados indivíduos com aproximadamente nove meses de idade, referidos neste trabalho como "juvenis". Estes foram obtidos no Viveiro Municipal da Ilha Comprida-SP, onde a semeadura e o crescimento inicial ocorreram em substrato composto de $60 \%$ de turfa, $20 \%$ de palha de arroz e $20 \%$ de vermiculita, em casa de vegetação. Quando as mudas atingiram aproximadamente 3 $\mathrm{cm}$ de altura, foi aplicado o adubo de superfície Polyon NPK 19:6:13, sendo 3 gramas por tubete, repetida a cada quatro meses. Quando atingiram alturas de aproximadamente $25 \mathrm{~cm}$, as mudas foram transplantadas para sacos plásticos pretos de 1,5 L preenchidos com substrato composto de $15 \%$ de vermiculita, $15 \%$ de palha de arroz, $40 \%$ de turfa e $30 \%$ de solo da restinga, sendo mantidas em pleno sol com irrigação a cada dois dias (Carrasco 2003).

No início do experimento, nove plântulas e cinco juvenis foram transplantados, em fevereiro de 2004 e novembro de 2003 respectivamente (inicio do perído de maior pluviosidade), diretamente no solo de cada ponto experimental, com espaçamento de $0,3 \mathrm{~m}$ para plântulas e $1 \mathrm{~m}$ para juvenis, sem a adição de adubo ou irrigação.

\section{Coleta de dados}

Para cada microambiente (SS, SU, CS e CU) foram selecionadas cinco sub-parcelas $(20 \times 20 \mathrm{~m})$ distintas, somando 20 pontos amostrais. A estimativa mensal (Novembro/2003 a Fevereiro/2005) da umidade do solo (percentual de água contido no peso total do substrato) foi realizada por meio do método gravimétrico (Embrapa 1997). Em cada ponto de amostragem foram coletadas três porções de solo de $0-5 \mathrm{~cm}$ de profundidade, compondo-se uma amostra que foi armazenada em recipiente plástico, imediatamente vedado com tampa e fita crepe. No laboratório, cada amostra foi pesada em balança de precisão e seca em estufa a $100^{\circ} \mathrm{C}$ até peso constante. Uma amostra de solo em cada ponto de amostragem também foi coletada para fins de análise química, realizada pelo Laboratório de Análise de Solo e Planta, da Universidade Federal de São Carlos (UFSCar), em Araras-SP.

A radiação fotossinteticamente ativa (RFA) ao nível do solo foi estimada, em dia de céu aberto, com poucas nuvens, entre 10:30 e 14:00h, em Fevereiro de 2005, período de maior luminosidade do ano. Foram realizadas, em cada ponto de amostragem, três medidas de luz obtidas por radiômetro LI 1000 (LiCOR) para obtenção da média local.

\section{Variáveis biológicas avaliadas}

As médias de peso seco e área foliar iniciais foram obtidas a partir de dez plântulas e cinco juvenis. $\mathrm{Na}$ avaliação após três meses de experimento foram utilizadas duas plântulas e um juvenil por ponto de amostragem, totalizando dez plântulas e cinco juvenis por ambiente, e, após um ano, todos os indivíduos sobreviventes foram avaliados. A área foliar foi estimada a partir da relação $\left(\mathrm{R}^{2}=0,98\right)$ entre a área "real" $(\mathrm{A})$ e o produto do comprimento do eixo longitudinal do limbo pela perpendicular ao eixo no ponto mais largo da folha (Barros et al. 1973). A área foliar real "A" foi obtida pelo recorte dos contornos das 
lâminas foliares delineadas em papel, e pesagem de cada corte em balança analítica, estimando-se $\mathrm{a}$ área com referência à massa da respectiva folha de papel (M) de área conhecida $\mathrm{F}$, de acordo com a fórmula: $\mathrm{A}=(\mathrm{F} \cdot \mathrm{m}) / \mathrm{M}$, onde $\mathrm{m}=$ massa do recorte de cada impressão foliar.

A Taxa de Crescimento Relativo (TCR), Taxa de Assimilação Líquida (TAL), Área Foliar Especifica (AFE), Razão de Área Foliar (RAF) e Razão de Massa Foliar (RMF) foram calculadas segundo Hunt (1982):

$\mathrm{TCR}=\ln \mathrm{M}_{2}-\ln \mathrm{M}_{1} / \mathrm{t}_{2}-\mathrm{t}_{1}$ (g.g. ${ }^{-1} \cdot$ mês $\left.^{-1}\right) \Rightarrow$ taxa de crescimento em relação ao tamanho do sistema

$\mathrm{TAL}=\mathrm{M}_{2}-\mathrm{M}_{1} / \mathrm{t}_{2}-\mathrm{t}_{1} \times \ln \mathrm{A}_{2}-\ln \mathrm{A}_{1} / \mathrm{A}_{2}-\mathrm{A}_{1}\left(\mathrm{~g} \cdot \mathrm{cm}^{-1}\right.$. mês $\left.{ }^{-1}\right) \Rightarrow$ eficiência do sistema assimilatório, onde: $\mathrm{M}_{1}$ $=$ massa da matéria seca total no tempo $\mathrm{t}_{1} ; \mathrm{M}_{2}=$ massa da matéria seca total no tempo $\mathrm{t}_{2} ; \mathrm{A}_{1}=$ área foliar total no tempo $\mathrm{t}_{1} ; \mathrm{A}_{2}=$ área foliar total no tempo $\mathrm{t}_{2} ; \mathrm{t}_{1}=$ tempo da primeira coleta; $t_{2}=$ tempo da segunda coleta.

A mesma fórmula de TCR foi utilizada para a estimativa da taxa de crescimento relativo da altura $\left(\mathrm{TCR}_{\text {alt }}\right)$ e do número de folhas $\left(\mathrm{TCR}_{n f}\right)$ os quais foram utilizados para análises estatísticas de correlação.

$\mathrm{AFE}=\mathrm{A}_{\text {folhas }} / \mathrm{MS}_{\text {follas }}\left(\mathrm{cm}^{2} \cdot \mathrm{g}^{-1}\right) \Rightarrow$ indicador da espessura relativa da folha;

$\mathrm{RAF}=\mathrm{A}_{\text {folhas }} / \mathrm{MS}_{\text {total }}\left(\mathrm{cm}^{2} \cdot \mathrm{g}^{-1}\right) \Rightarrow$ dimensão relativa do aparelho fotossintético;

$\mathrm{RMF}=\mathrm{MS}_{\text {folhas }} / \mathrm{MS}_{\text {total }}\left(\mathrm{g} \cdot \mathrm{g}^{-1}\right) \Rightarrow$ fração da biomassa alocada para as folhas, onde: $\mathrm{A}=$ área foliar; $\mathrm{MS}$ = matéria seca.

\section{Análises estatísticas}

As taxas de sobrevivência e crescimento em altura ao longo de um ano foram estimadas a partir das inclinações das curvas de regressão linear e comparadas por intermédio do Teste T. Para a comparação de médias das variáveis morfofisiológicas, foram realizadas análises de variância (ANOVA) seguidas de teste de Tukey, ou, quando os dados revelaram heterocedasticidade, análise de variância de Kruskall-Wallis (Zar 1996). Para a correlação entre os dados obtidos ao final do experimento de campo com os dados físicos (luz, nutrientes, e média anual do percentual de umidade do solo), foi utilizado o teste de Pearson, em nível de significância de 5\%.

\section{Resultados}

Os valores estimados de luz e umidade do solo, e atributos químicos do solo estão apresentados nas Tabelas 1 e 2, respectivamente. Para calcular as médias anuais de umidade do solo (\%) foi considerado o período de Janeiro a Dezembro/2004, enquanto as mínimas e máximas mensais foram obtidas ao longo dos ensaios (novembro/2003 a fevereiro/2005). Os menores percentuais de umidade do solo foram obtidos em dezembro/2003, e os maiores, em fevereiro/2004, março/2004, janeiro/2005 e fevereiro/2005 para o SS, SU, CS e $\mathrm{CU}$, respectivamente. $\mathrm{O}$ ambiente de $\mathrm{CS}$ apresentou a maior radiação fotossinteticamente ativa e a maior oscilação mensal no teor de água do solo, enquanto o SU apresentou a menor incidência de luz e o menor valor de saturação de bases no solo, considerado um bom indicador de fertilidade. Em geral, os ambientes de maior umidade (SU e CU) se caracterizam por teores mais elevados de matéria orgânica, capacidade de troca catiônica e acidez potencial do solo quando comparados aos de solo mais secos, os quais exibiram maiores índices de fertilidade.

No período de doze meses a quantidade de plântulas vivas decresceu em todos os ambientes, sendo que o maior decréscimo ocorreu em SU (Teste T, $\alpha=0,05$ ) (Fig. 1a). Na correlação de matrizes (Pearson), a sobrevivência das plântulas de $O$. pulchella se relacionou significativamente $(\mathrm{p}<0,05)$ apenas às variáveis bióticas (Tab. 3).

Tabela 1 - Valores médios e erros-padrão de radiação fotossinteticamente ativa (RFA, $\mu$ mol. $\mathrm{m}^{-2} \cdot \mathrm{s}^{-1}$ ) estimados em Fevereiro de 2005, e percentuais de umidade do solo (Média anual, e médias mensais mínimas e máximas ao longo dos experimentos) nos diferentes microambientes de uma floresta de restinga da Ilha do Cardoso-SP. SS= sub-bosque seco; $\mathrm{SU}=$ sub-bosque úmido; $\mathrm{CS}=$ clareira seca; $\mathrm{CU}=$ clareira úmida.

Table 1 -Mean values and standard errors of light $\left(\mathrm{PAR}, \mu \mathrm{mol} \cdot \mathrm{m}^{-2} . \mathrm{s}^{-1}\right)$ obtained in February 2005, and, percentage soil moisture (average annual, and monthly mean minimum and maximum along the experiments), in different microenvironments of a restinga forest at Illha do Cardoso, SP. SS= dry understory; $\mathrm{SU}=$ wet understory; $\mathrm{CS}=$ dry gap; $\mathrm{CU}=$ wet gap.

\begin{tabular}{lcccc}
\hline & & \multicolumn{3}{c}{ Umidade do solo (\%) } \\
& $\begin{array}{c}\text { RFA } \\
\left(\mu \mathrm{mol} . \mathrm{m}^{-2} \cdot \mathrm{s}^{-1}\right)\end{array}$ & $\begin{array}{c}\text { Média } \\
\text { anual }\end{array}$ & $\begin{array}{c}\text { Min. } \\
\text { mensal }\end{array}$ & $\begin{array}{c}\text { Máx. } \\
\text { mensal }\end{array}$ \\
\hline SS & $43( \pm 15)$ & $25( \pm 2)$ & $21(5)$ & $36,5( \pm 6)$ \\
SU & $13( \pm 3)$ & $83( \pm 1)$ & $75(1)$ & $92( \pm 9)$ \\
CS & $561( \pm 99)$ & $29( \pm 2)$ & $9(1)$ & $38( \pm 7)$ \\
CU & $324( \pm 123)$ & $81( \pm 2)$ & $65(8)$ & $84( \pm 1)$ \\
\hline
\end{tabular}


Tabela 2 - Analise química do solo (valores médios) em diferentes microambientes de uma floresta de restinga da Ilha do Cardoso-SP. SS: sub-bosque seco; SU: sub-bosque úmido; CS: clareira seca; CU: clareira úmida. SB: soma de bases; CTC: capacidade de troca catiônica; V: percentagem de saturação de bases no solo.

Table 2 - Chemical analysis of soil (average values) in different microenvironments of a restinga forest at Ilha do Cardoso, SP. SS= dry understory; $\mathrm{SU}=$ wet understory; $\mathrm{CS}=$ dry gap; $\mathrm{CU}=$ wet gap. $\mathrm{SB}=$ sum of bases; $\mathrm{CTC}=$ cation exchange capacity; $\mathrm{V}$ : percentage of base saturation in soils.

\begin{tabular}{|c|c|c|c|c|c|c|c|c|c|c|c|}
\hline & $\mathrm{P}$ & $\mathrm{MO}$ & $\mathrm{pH}$ & K & $\mathrm{Ca}$ & $\mathrm{Mg}$ & $\mathrm{H}+\mathrm{Al}$ & $\mathrm{Al}$ & SB & CTC & $\mathrm{V}$ \\
\hline $\mathrm{mg} \cdot \mathrm{dm}^{-3}$ & & g. $\mathrm{dm}^{-3}$ & $\mathrm{CaCl}_{2}$ & & & & mol.dm & & & & $\%$ \\
\hline SS & 4,2 & 87,2 & 3,4 & 2,1 & 12,2 & 8,8 & 100,2 & 8,8 & 23,1 & 123,0 & 18,4 \\
\hline SU & 43,4 & 140,0 & 3,2 & 2,6 & 17,2 & 13,8 & 244,8 & 15,8 & 33,6 & 278,4 & 11,8 \\
\hline CS & 7,4 & 59,0 & 4,0 & 1,0 & 22,2 & 9,0 & 59,2 & 45,1 & 32,2 & 91,0 & 38,0 \\
\hline $\mathrm{CU}$ & 44,8 & 192,0 & 3,4 & 2,4 & 31,6 & 14,0 & 234,0 & 15,6 & 48,0 & 282,0 & 21,6 \\
\hline
\end{tabular}

Os valores (médias e erros-padrão) iniciais de altura e número de folhas das plântulas instaladas em campo foram, respectivamente, de $4,8 \mathrm{~cm}( \pm 0,2)$ e $6( \pm 0,2)$. Observou-se leve aumento da altura das plântulas em todos os ambientes. Entretanto as taxas de crescimento em altura, estimadas a partir das inclinações das curvas de regressão linear, foram maiores em indivíduos mantidos em clareiras do que em sub-bosque (Teste T, $\alpha=0,05$ ) (Fig. 2a). Após três meses, não foi observada variação da massa seca de raízes e parte aérea em comparação com os valores iniciais em nenhum dos ambientes, entretanto, após 12 meses houve um aumento ( $\mathrm{p}<$ 0,05 , teste de Tukey) da massa seca da raiz e parte aérea apenas nos ambientes de clareira (CS e CU) (Fig. 3a). Após um ano, houve um aumento da área foliar (AF) em comparação com o tempo zero nas plântulas mantidas em clareira $(\mathrm{p}<0,001)$ (Fig. 4a). As variáveis RAF e AFE (Fig. 4c e 4g) aumentaram independentemente do ambiente, enquanto que a RMF não diferiu em relação ao tempo zero em nenhum deles (Fig 4e). As taxas de assimilação líquida (TAL), em mg.cm-2 $\mathrm{mês}^{-1}$, de plântulas mantidas em CS (TAL $=0,9 \pm 0,3)$ e CU $(0,7 \pm$ $0,3)$ apresentaram variação positiva ao final de 12 meses, não se observando variação em plantas de SS $(-0,2 \pm 0,2)$. No SU ocorreu variação negativa da TAL (-0,8 mg.cm $\left.{ }^{-2} \cdot \mathrm{mês}^{-1} \pm 0,3\right)$, variação essa significativamente menor do que a observada nas plântulas mantidas em clareiras. Observou-se um efeito da luz (correlação de Pearson, $\mathrm{p}<0,05$ ) sobre diversas variáveis de crescimento, enquanto a umidade do solo não foi relacionada a nenhuma delas, enquanto que a TCRalt correlacionou-se a alguns atributos químicos do solo (Tab. 3).
Tabela 3 - Resultado da correlação de Pearson obtido com os dados de fatores abióticos, sobrevivência e variáveis de crescimento, estimadas ao final de um ano de experimento, em plântulas de Ocotea pulchella em diferentes microambientes de uma floresta de Restinga da Ilha do Cardoso, SP.

Table 3 - Results of Pearson correlation with data obtained from abiotic factors, survival and growth variables, estimated at the end of a year of experiment, in seedlings of Ocotea pulchella in different microenvironments of a restinga forest at Ilha do Cardoso, SP.

\begin{tabular}{lcccccc}
\hline & Sobrev. & $\mathbf{T C R} \boldsymbol{m}$ & $\mathbf{L u z}$ & $\mathbf{p H}$ & $\mathbf{K}$ & $\mathbf{V}$ \\
\hline TCRalt & 0,60 & 0,75 & 0,67 & 0,44 & $-0,53$ & 0,45 \\
$\mathrm{TCR} n f$ & $*$ & 0,48 & 0,72 & $*$ & $*$ & $*$ \\
$\mathrm{TCR} m$ & 0,54 & & 0,61 & $*$ & $*$ & $*$ \\
$\mathrm{AF}$ & $*$ & 0,71 & 0,58 & $*$ & $*$ & $*$ \\
$\mathrm{AFE}$ & $*$ & $-0,53$ & $*$ & $*$ & $*$ & $*$ \\
$\mathrm{RMF}$ & $*$ & 0,51 & 0,52 & $*$ & $*$ & $*$ \\
$\mathrm{TAL}$ & $*$ & 0,68 & 0,61 & $*$ & $*$ & $*$
\end{tabular}

Sobrev. $=$ Percentagem de sobrevivência de plântulas. TCRalt= Taxa de crescimento relativo em altura; TCR $n f=$ Taxa de crescimento relativo em número de folhas; TCR $m=$ Taxa de crescimento relativo em massa seca; $\mathrm{AF}=$ Área foliar; $\mathrm{AFE}=$ Área foliar específica; $\mathrm{RMF}=$ Razão de massa foliar; TAL = Taxa de assimilação líquida; Luz= dados de radiação fotossinteticamente ativa $\left(\mathrm{em} \mu \mathrm{mol} \cdot \mathrm{m}^{-2} \cdot \mathrm{s}^{-1}\right)$; Umi= Percentagem de umidade do solo (média anual); $\mathrm{V}=$ percentagem de saturação de bases no solo.

Sobrev: Percentage of seedling survival. TCRalt= relative growth rate in height; TCRnf= relative growth rate in number of leaves; TCR $m=$ relative growth rate in dry mass; $\mathrm{AF}=\mathrm{Leaf}$ area; $\mathrm{AFE}=$ specific leaf area; $\mathrm{RMF}=$ leaf mass ratio; $\mathrm{TAL}=$ net assimilation rate; $\mathrm{Light}=$ data photosynthetically active radiation (in $\mu \mathrm{mol} \cdot \mathrm{m}^{-2} . \mathrm{s}^{-1}$ ); Umi= percentage of soil moisture (annual mean); $\mathrm{V}=$ percentage of base saturation in the soil. $\mathrm{CTC}=$ cation exchange capacity; $\mathrm{V}=$ percentage of base saturation in soils. 

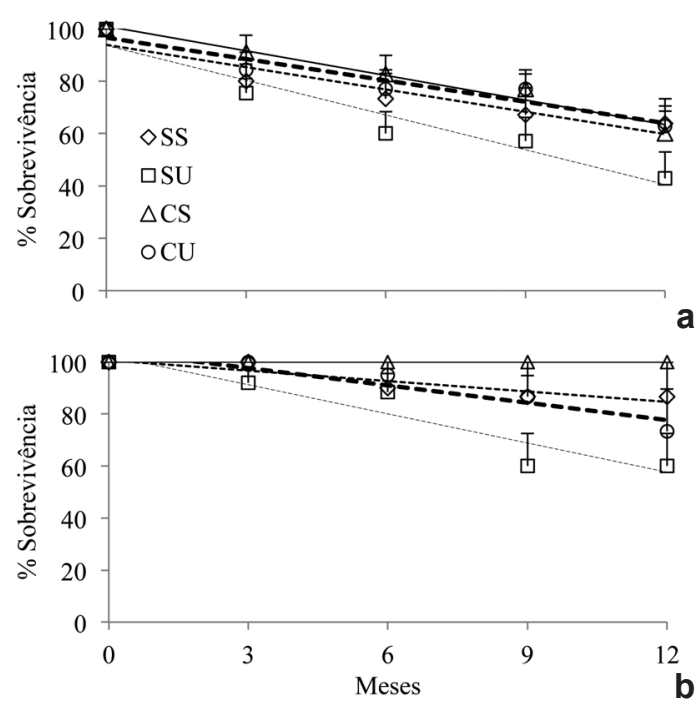

Figura 1 -Percentuais de sobrevivência $( \pm \mathrm{EP})$ de plântulas (a) ejuvenis (b) de Ocotea pulchella, mantidas em sub-bosque $\operatorname{seco}\left(\diamond-\mathrm{SS} ; \mathrm{Y}_{\text {plântulas }}=93,9-2,84 \mathrm{x} ; \mathrm{Y}_{\text {juvenis }}=100,67-1,33 \mathrm{x}\right)$; sub-bosque úmido ( $\square-\mathrm{SU}$; $\mathrm{Y}_{\text {plântulas }}=93,65-4,42 \mathrm{x}$; $\mathrm{Y}_{\text {juvenis }}$ $=102,46-3,73 x)$; clareira seca $\left(\Delta-C S, Y_{\text {plântulas }}=101,01\right.$ $-3,13 x)$ e clareira úmida ( $\circ-C U$; $Y_{\text {plântulas }}=96,63-4,42 x$; $\left.\mathrm{Y}_{\text {juvenis }}=104,33-2,22 \mathrm{x}\right)$.

Figure 1 - Survival rates ( \pm SE) of seedlings (a) and juveniles (b) of Ocotea pulchella, maintained in dry understory $\left(\diamond-\mathrm{SS}, \mathrm{Y}_{\text {seddlings }}-2.84\right.$ $\left.\mathrm{x}=93.9 ; \mathrm{Y}_{\text {juveniles }}-1=100.67,33 \mathrm{x}\right)$; moist understory $\left(\square-\mathrm{SU} ; \mathrm{Y}_{\text {seedlings }}\right.$ $\left.=93.65 \mathrm{x}-4.42 ; \mathrm{Y}_{\text {juveniles }}=102.46-3.73 \mathrm{x}\right)$, dry gap $\left(\Delta-\mathrm{CS}, \mathrm{Y}_{\text {seedlings }}=\right.$ $101.01-3,13 \mathrm{x})$ and wet gap $\left(\mathrm{O}-\mathrm{CU} ; \mathrm{Y}_{\text {seedlings }}=96.63 \mathrm{x}-4.42 ; \mathrm{Y}_{\text {juveniles }}\right.$ $=104.33-2.22 \mathrm{x})$.

Nos juvenis, a quantidade de plantas vivas em CS permaneceu constante ao longo dos doze meses do experimento, enquanto nos demais ambientes houve um decréscimo a partir do terceiro mês, sendo a maior mortalidade verificada no SU, seguida das CU e SS (Teste T, $\alpha=0,05$ ). A sobrevivência relacionou-se $(\mathrm{p}<0,05)$ a diversas variáveis ambientais (umidade e atributos químicos do solo), mas apenas a uma variável biótica (número inicial de folhas) $(0,47)$ (Tab. 4). A taxa de crescimento em altura dos juvenis foi menor (Teste $\mathrm{T}, \alpha=0,05$ ) em SU do que nos demais ambientes, os quais não diferiram entre si, ressaltando-se que em SU não houve incremento significativo em relação à altura média dos indivíduos no início do experimento (Fig. 2b). Em um ano não ocorreu qualquer acréscimo significativo da massa seca (raiz e parte aérea) em relação ao tempo zero em nenhum dos tratamentos, assim como não houve diferenças significativas entre os ambientes (Fig. 3b).

Nos juvenis, os valores de AF (Fig 4b) e RAF (Fig. 4h) permaneceram estáveis ao longo do período

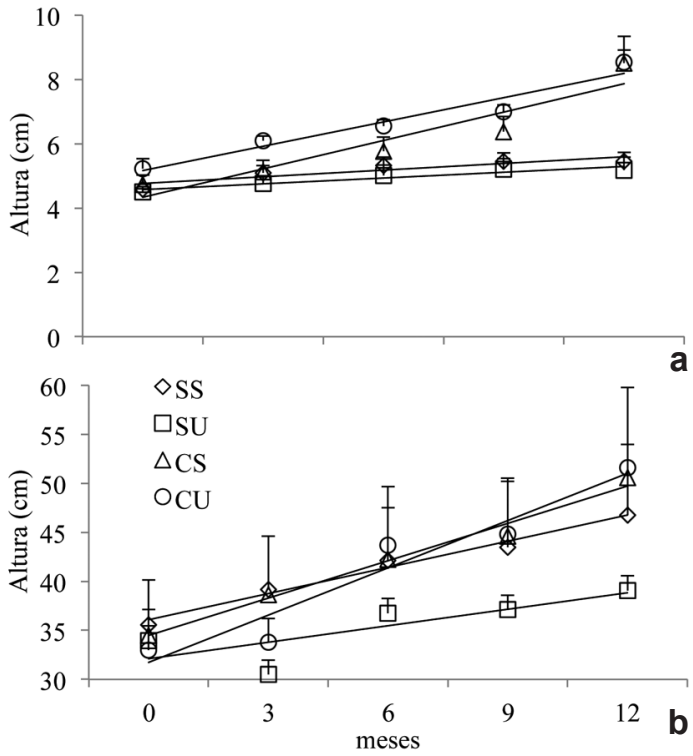

Figura 2 - Médias de altura da parte aérea de plântulas (a) e juvenis (b) de Ocotea pulchella, mantidas em sub-bosque $\operatorname{seco}\left(\diamond-\mathrm{SS} ; \mathrm{Y}_{\text {plântulas }}=4,76+0,07 \mathrm{x} ; \mathrm{Y}_{\text {juvenis }}=36,08+0,89 \mathrm{x}\right)$; sub-bosque úmido ( $\square-\mathrm{SU} ; \mathrm{Y}_{\text {plântulas }}=4,57+0,06 \mathrm{x}$; $\mathrm{Y}_{\text {juvenis }}=$ $32,1+0,56 x)$; clareira seca $\left(\Delta-C S, Y_{\text {plântulas }}=4,33+0,29 x\right.$; $\left.\mathrm{Y}_{\text {juvenis }}=34,48+1,27 \mathrm{x}\right)$ e clareira úmida $\left(\mathrm{O}-\mathrm{CU}\right.$; $\mathrm{Y}_{\text {plântulas }}=$ $\left.5,17+0,25 \mathrm{x} ; \mathrm{Y}_{\text {juvenis }}=31,73+1,61 \mathrm{x}\right)$.

Figure 2 - Height growth rates of shoots (in log scale) of seedlings (a) and juveniles (b) of Ocotea pulchella, maintained in dry understory $-\mathrm{SS}\left(\diamond-\mathrm{SS}, \mathrm{Y}_{\text {seedlings }}=4.76+0.07 \mathrm{x} ; \mathrm{Y}_{\text {juveniles }}=36.08+\right.$ $0.89 \mathrm{x})$; moist understory $-\mathrm{SU}\left(\square-\mathrm{SU} ; \mathrm{Y}_{\text {sedlings }}=4.57+0.06 \mathrm{x}\right.$; $\left.\mathrm{Y}_{\text {juveniles }}=32.1+0.56 \mathrm{x}\right)$, dry gap $-\mathrm{CS}\left(\Delta-\mathrm{CS}, \mathrm{Y}_{\text {seedlings }}=4.33+0.29\right.$ $\left.\mathrm{x} ; \mathrm{Y}_{\text {juveniles }}=34.48+1.27 \mathrm{x}\right)$ and wet gap $\left(\mathrm{O}-\mathrm{CU} ; \mathrm{Y}_{\text {seedlings }}=5.17+\right.$ $\left.0.25 \mathrm{x} ; \mathrm{Y}_{\text {juveniles }}=31.73+1.61 \mathrm{x}\right)$.

de observações nos ambientes SS, CS e CU, ocorrendo diminuição significativa (Teste de Tukey, $\mathrm{p}<0,001$ ) em plantas mantidas em SU (Fig. 4b). Os valores de AFE e RMF não variaram significativamente em relação ao início do experimento em nenhum dos ambientes (Fig. 4d e 4f). A estimativa da média anual da Taxa de Assimilação Líquida, em mg.cm ${ }^{-2}$. ês $^{-1}$, dos juvenis em SU $(-2,5 \pm 0,7)$ foi significativamente menor do que nos demais ambientes ( $\mathrm{SS}=-0,2 \pm 0,6$; $\mathrm{CS}=0,1 \pm 0,4$; e CU $=0,2 \mathrm{mg} \cdot \mathrm{cm}^{-2} \cdot \mathrm{mês}^{-1} \pm 0,5$ ).

As análises estatísticas de correlação (Pearson) indicam um efeito da luz $(\mathrm{p}<0,05)$ sobre as diversas variáveis de crescimento, mas nenhuma correlação dessas com a umidade e características químicas do solo (Tab. 4).

\section{Discussão}

Com exceção dos juvenis mantidos em CS, onde não se registrou mortalidade de indivíduos, 
nos demais ambientes houve um decréscimo no percentual de sobrevivência, tanto de juvenis como de plântulas. O SU foi o ambiente onde se observou as maiores taxas de mortalidade em ambos os grupos de idade, provavelmente devido a fatores fisiológicos, já que não foram observados sinais de ação intensa de herbívoros. Por outro lado, notouse a presença ocasional de hifas de fungos sobre a superfície de folhas de plântulas mantidas em SU.

Nas plântulas a sobrevivência não foi relacionada diretamente aos fatores abióticos, mas aos parâmetros de crescimento, os quais por sua vez foram favorecidos em clareiras, assim como ocorre em diversas espécies arbóreas tropicais (Pompa \& Bongers 1988; Yan \& Cao 2008). Além da luz, a maior fertilidade do solo $(\% \mathrm{~V})$ nas clareiras, especialmente as secas, também pode ter influenciado o maior crescimento em altura nos ambientes com maiores irradiâncias, já que as variáveis "fertilidade" e "crescimento em altura" foram correlacionadas.

Nos juvenis de $O$. pulchella a sobrevivência correlacionou-se aos atributos edáficos associados à alta umidade do solo, mas não às variáveis bióticas, como a TCR, o que poderia indicar que os indivíduos nesta fase mantem maior canalização de recursos para processos voltados à manutenção do que ao crescimento (Grime 1977), permitindo ao indivíduo sobreviver por longos períodos no sub-bosque da floresta, mesmo sem crescer. Todavia, os juvenis mantidos em SU, além de menor sobrevivência, também apresentaram menor crescimento em altura, área foliar e a taxa de assimilação líquida do que nos demais ambientes. Neste ambiente, embora não ocorra inundação permanente, a influência da alta saturação hídrica é evidente na espessa camada de serapilheira mantida graças à baixa aeração do solo que reduz as taxas de decomposição da matéria orgânica (Gomes et al. 2007). Desta forma, a hipóxia modifica o balanço de nutrientes minerais, ocasionando maior saturação por alumínio e baixo $\mathrm{pH}$ do solo, o que pode reduzir a absorção e transporte de íons e levar a planta a um estresse nutricional (Kozlowski et al. 1991). A resposta de plantas jovens de $O$. pulchella à alta umidade do solo pode ser ilustrada em experimento realizado exsitu em condições semi-controladas, no qual juvenis desta espécie apresentaram redução significativa do crescimento em substrato alagado em diferentes condições de luz (Pires \& Cardoso 2011).

A sensibilidade aparentemente maior de juvenis de $O$. pulchella às condições de alta saturação hídrica do solo em sub-bosque do que em clareiras, pode estar associada, em parte, aos valores
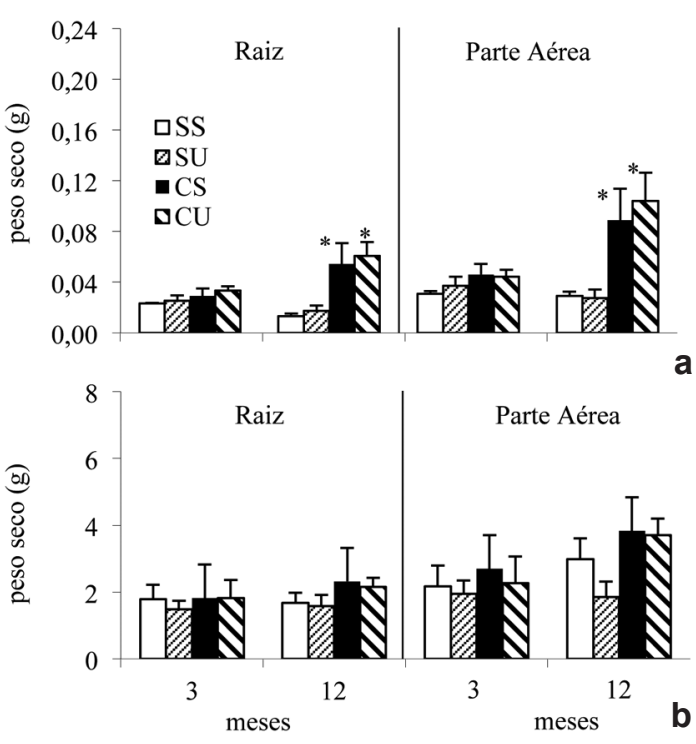

Figura 3 - Peso seco $( \pm$ EP) da raiz e parte aérea de plântulas (a) e juvenis (b) de Ocotea pulchella, após 3 e 12 meses em sub-bosque seco (SS); sub-bosque úmido (SU); clareira seca (CS) e clareira úmida (CU), numa floresta de Restinga. Médias iniciais (tempo zero) de plântulas (a): 0,03g de raiz, e 0,04g de parte aérea; e de Juvenis (b): 2,7g de raiz, e, 2,6g de parte aérea. *=Valor difere significativamente da média inicial $(\mathrm{p}<0,05)$.

Figure 3 - Dry weight $( \pm$ SE) of roots and shoots of seedlings (a) and juveniles (b) of Ocotea pulchella, after 3 and 12 months in dry understory (SS), moist understory (SU), dry gap (CS) and wet gap (CU) in a restinga forest. Initial (time zero) root and shoot dry weight are $0.03 \mathrm{~g}$ and $0.04 \mathrm{~g}$, respectively, for seedlings (a); and $2.7 \mathrm{~g}$ and $2.6 \mathrm{~g}$ for juveniles (b). * = Value differs significantly from the average initial $(\mathrm{p}<0,05)$.

mais elevados de fertilidade nestas últimas, bem como à menor capacidade da planta em absorver $\mathrm{e}$ reter água e nutrientes no ambiente mais sombreado, como sugerido por Kramer \& Kozlowski (1979) e Smith \& Huston (1989). Por sua vez, o maior desenvolvimento (por ex, maior crescimento em altura, área foliar e taxa de assimilação líquida) dos juvenis de $O$. pulchella em SS em comparação com o $\mathrm{SU}$, não pode ser atribuído unicamente às condições do solo, mas também a maior radiação fotossintenticamente ativa (e possivelmente maiores razões vermelho:vermelho extremo) que atinge o nível do solo em SS, já que o dossel, em geral, é mais aberto, e a luz foi positivamente correlacionada ao parâmetros de crescimentos.

Os valores de AF, RAF (área foliar:massa planta) e AFE (área foliar:massa foliar) aumentaram em plântulas de $O$. pulchella nos quatro microambientes 

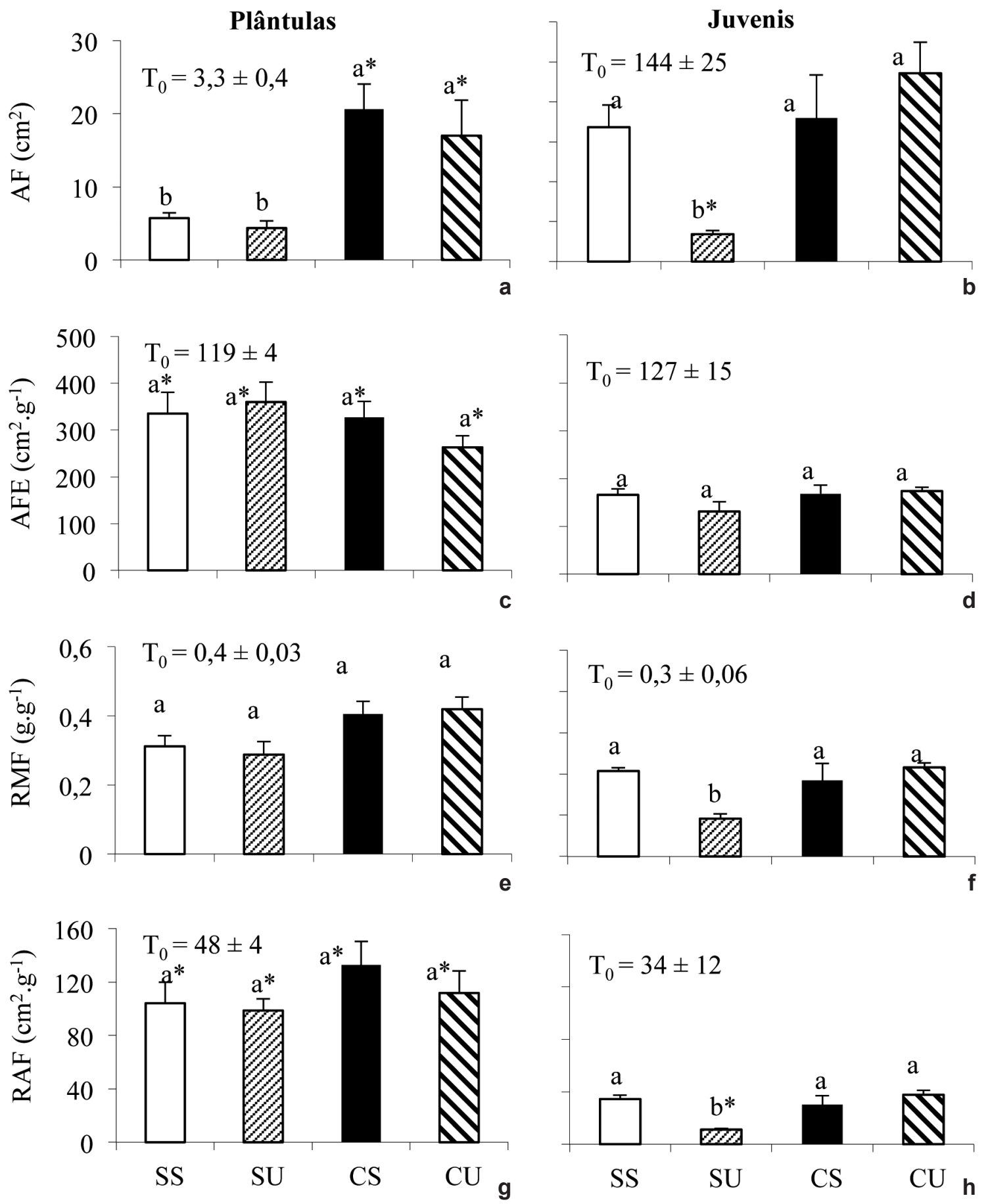

Figura 4 - Área Foliar (a, b), Área Foliar Específica (c, d), Razão de Massa Foliar (e, f) e Razão de Área Foliar (g, h) (médias \pm EP) de plântulas (coluna da esquerda) e juvenis (coluna da direita) de Ocotea pulchella após 12 meses em quatro ambientes (sub-bosque seco, SS; sub-bosque úmido, SU; clareira seca, CS; e clareira úmida, CU) em floresta de restinga. Médias seguidas da mesma letra não diferem entre si ( $<<0,05$, teste de Tukey). *= valor difere significativamente da média inicial $(\mathrm{p}<0,05)$.

Figure 4 - Leaf area (a, b), specific leaf area (c, d), leaf weight ratio (e, f) and leaf area ratio ( $g, h)$ (mean \pm SE) of seedlings (left column) and juveniles (right column) of Ocotea pulchella after 12 months in four environments (dry understory, SS; moist understory, SU, dry gap, $\mathrm{CS}$, and wet gap, $\mathrm{CU}$ ) in the restinga forest. Means followed by same letter do not differ ( $\mathrm{p}<0.05$, Tukey test). * = value differs significantly from the average initial $(\mathrm{p}<0,05)$. 
Tabela 4 - Resultado da correlação de Pearson obtido com os dados de fatores abióticos, sobrevivência e variáveis de crescimento, estimadas ao final de um ano de experimento, de juvenis de Ocotea pulchella em diferentes microambientes de uma floresta de restinga da Ilha do Cardoso, SP.

Table 4 - Results of Pearson correlation with data obtained from abiotic factors, survival and growth variables, estimated at the end of a year of experiment, in juveniles of Ocotea pulchella in different microenvironments of a restinga forest at Ilha do Cardoso, SP.

\begin{tabular}{cccccccc}
\hline & TCRm & Luz & Umi & pH & CTC & H+Al & V \\
\hline Sobreviv. & $*$ & $*$ & $-0,50$ & 0,57 & $-0,58$ & $-0,63$ & 0,66 \\
TCRalt & 0,64 & 0,60 & $*$ & $*$ & $*$ & $*$ & $*$ \\
TCRnf & 0,63 & 0,64 & $*$ & $*$ & $*$ & $*$ & $*$ \\
$\mathrm{TCR} m$ & $*$ & 0,60 & $*$ & $*$ & $*$ & $*$ & $*$ \\
$\mathrm{AF}$ & 0,61 & 0,56 & $*$ & $*$ & $*$ & $*$ & $*$ \\
$\mathrm{RMF}$ & $*$ & 0,50 & $*$ & $*$ & $*$ & $*$ & $*$ \\
$\mathrm{TAL}$ & 0,82 & 0,62 & $*$ & $*$ & $*$ & $*$ & $*$ \\
\hline
\end{tabular}

Sobrev.: Percentagem de sobrevivência de juvenis. TCRalt: Taxa de crescimento relativo em altura; TCRnf: Taxa de crescimento relativo em número de folhas; TCRm: Taxa de crescimento relativo em massa seca; AF: Área foliar; RMF: Razão de massa foliar; TAL: Taxa de assimilação líquida. Luz: dados (em $\mu$ mol. $\mathrm{m}^{-2} \cdot \mathrm{s}^{-1}$ ) coletados em Fevereiro de 2005; Umi: Percentagem de umidade do solo (média anual); $\mathrm{pH}$ : potencial hidrogeniônico; $\mathrm{CTC}$ : capacidade de troca catiônica; e V: percentagem de saturação de bases no solo.

Sobrev $=$ Percentage of seedling survival. TCRalt = relative growth rate in height; $\mathrm{TCR} n f=$ relative growth rate in number of leaves; $\mathrm{TCR} m=$ relative growth rate in dry mass; $\mathrm{AF}=$ Leaf area; $\mathrm{RMF}=$ leaf mass ratio; $\mathrm{TAL}=$ net assimilation rate; Light $=$ data photosynthetically active radiation (in $\mu \mathrm{mol} \cdot \mathrm{m}^{-2} \cdot \mathrm{s}^{-1}$ ); Umi= percentage of soil moisture (annual average); $\mathrm{V}=$ percentage of base saturation in the soil; $\mathrm{pH}=$ hydrogen potential; $\mathrm{CTC}=$ cation exchange capacity; $\mathrm{V}=$ percentage of base saturation in soils.

estudados, enquanto que a RMF (massa foliar:massa total) não variou, ou oscilou negativamente, em relação ao início tempo zero, indicando uma maior expansão da área foliar relativa à massa. Vale ressaltar que, nas plântulas, a área foliar variou positivamente, especialmente nas clareiras, embora tenha ocorrido redução no número de folhas. Em juvenis, por outro lado, não houve crescimento significativo após um ano em relação a nenhum dos quatro parâmetros, chegando a ocorrer uma redução nas plantas de SU. O baixo investimento em aparato fotossintético pode representar uma vantagem para os indivíduos submetidos às condições adversas (Poorter 1999; Souza \& Válio 2003) comumente encontradas na restinga, cerrado e floresta estacional, possivelmente contribuindo para o sucesso da espécie em colonizar tais ambientes. Outra característica atribuída às plantas que crescem em solos oligotróficos ou em vegetação em estágio inicial de sucessão é a presença de folhas com alto grau de esclerofilia, que pode ser avaliado por intermédio da AFE (Camerik \& Werger 1981; Marin \& Medina 1981). Em O. pulchella, as folhas das plântulas apresentaram-se pouco espessas (altos valores de AFE), comparáveis aos de espécies florestais submetidas a diferentes condições de luz e espécies de cerrado sob condição de baixa luminosidade (Hoffman \& Franco 2003). Entretanto, é provável que a esclerofilia aumente com a idade da planta de $O$. pulchella, considerando-se os baixos valores de AFE encontrados no juvenis avaliados neste trabalho, bem como em indivíduos adultos desta espécie (Boeger \& Wisniewski 2003).

As taxas de assimilação líquida (TAL) negativas de $O$. pulchella no sub-bosque estiveram associadas principalmente à perda de massa seca total no caso das plântulas. No caso dos juvenis, a variação negativa de TAL em SU ocorreu em função do declínio - ainda que não significativo - da massa, bem como da perda de área foliar decorrente da queda de folhas. Em SS, assim como nas clareiras, os juvenis apresentaram variação positiva da área foliar e demais parâmetros associados. Todavia, o valor de TAL não foi positivo, como observado nos indivíduos mantidos em clareiras, os quais apresentaram também maior valor de TCR. Os resultados sugerem uma descontinuidade entre as variáveis massa e área foliar, ou seja, o crescimento de ambas não segue o mesmo expoente e nem varia de modo contínuo no intervalo de tempo considerado (Magalhães 1979).

Neste trabalho, observaram-se correlações mais fortes das TCR (massa seca) das plantas de $O$. pulchella com as TAL - que indicam a 'eficiência' do sistema assimilatório - do que com a RAF ou AFE, assim como demonstrado por outras espécies de plantas crescendo em alta irradiação (Shipley 2002, 2006).

A variação negativa de parâmetros do crescimento sugere que $O$. pulchella pode sobreviver, pelo menos por algum tempo, em ambientes com pouca iluminação e elevada saturação hídrica no solo. Alterações ambientais, como a abertura de clareiras, poderiam aumentar a disponibilidade de luz e favorecer a fotossíntese líquida, tal como descrito para algumas espécies tardias da sucessão (Souza \& Válio 2003). As correlações positivas observadas entre as variáveis de crescimento e luz, também podem indicar uma dependência da espécie em relação a ambientes mais iluminados ou pequenas clareiras, o que explicaria a presença da espécie em fisionomias mais abertas, como as restingas e cerrados (Sugiyama 1998; Pinheiro \& 
Monteiro 2006), em detrimento da ocorrência em florestas mais fechadas (p. ex. floresta ombrófila densa). Esta característica deve ter sido determinante na classificação de $O$. pulchella como espécie do estágio inicial de sucessão (Salimon \& Negrelle2001; Carrasco 2003). Por outro lado, O. pulchella apresenta algumas características opostas ao descrito para tais espécies, como crescimento relativamente lento e pouco investimento em biomassa foliar, aqui reportados, bem como sementes sem dormência capazes de germinar na sombra (Pires et al. 2009).

O reduzido crescimento de plântulas e juvenis de $O$. pulchella deve estar associado ao caráter oligotrófico da restinga, a exemplo do que já foi relatado para outras espécies neste ecossistema (Zamith \& Scarano 2006; Fidalgo et al. 2009). As pequenas taxas de crescimento, especialmente nos juvenis e em plantas mantidas no sub-bosque, são corroboradas por estudos complementares sobre a regeneração natural de $O$. pulchella realizados ao longo de um ano nos mesmos ambientes, os quais apontam que o crescimento de plântulas ocorre apenas em indivíduos que emergem em clareiras (Pires 2006).

O comportamento de certa forma generalista exibido pelas plântulas e juvenis de $O$. pulchella deve possibilitar a colonização de distintos microambientes da restinga. No entanto, nas presentes condições experimentais, os resultados sugerem que baixas irradiâncias no sub-bosque quando associadas a solos saturados e quimicamente pobres podem atuar como fator limitante para o crescimento dos juvenis, o que pode levar a uma menor competitividade da espécie em relação àquelas mais adaptadas a essas condições. Isto é corroborado pela distribuição dos indivíduos arbóreos e plântulas de $O$. pulchella na parcela de restinga, os quais tenderam a apresentar menor densidade nos ambientes caracterizados como SU (Pires 2006). Os resultados podem ajudar a explicar a distribuição em manchas da população dos indivíduos arbóreos de O. pulchella na área de estudo obtida por Lima et al. (2011), e reforça a ideia de que o recrutamento da espécie pode ser limitado em formações florestais de restingas situadas em solo muito úmido.

\section{Agradecimentos}

Ao CNPq, a bolsa de doutorado concedida a primeira autora e auxilio financeiro (Processo: 473005/2003-4); ao Projeto "Diversidade, Dinâmica e Conservação em Florestas do Estado de São Paulo: 40 ha de Parcelas Permanentes" (Biota/Fapesp Processo: 1999/09635-0) o apoio logístico; ao Viveiro Municipal de Ilha Comprida, na pessoa de Pablo Carrasco, a concessão das mudas; a Ednilson da Silva, Cláudio Bernardes e Antônio de Pádua o auxílio no campo.

\section{Referências}

Araujo, D.S.D. \& Henriques, R.B.P. 1984. Análise florística das restingas do estado do Rio de Janeiro. In: Lacerda, L.D.; Araujo, D.S.D.; Cerqueira, R. \& Turcq, B. (eds.). Restingas: origem, estrutura, processos. Universidade Federal Fluminense/ CEUFF, Niterói, Pp. 159-193.

Augspurger, C.K. 1984. Light requeriments of neotropical tree seedlings: a comparative study of growth and survival. Journal of Ecology 72: 777-795.

Barros, R.S.; Maestri, M.; Vieira, M.; Braga-Filho, L.J. 1973. Determinação de área de folhas do café (Coffea arabica L. cv. 'Bourbon Amarelo'). Revista Ceres 20: 44-52.

Bazzaz, F.A. \& Pickett, S.T.A. 1980. Physiological ecology of tropical succession: a comparative review. Annual review of ecology and systematics 11: 287-310.

Boeger, M.R.T. \& Wisniewski, C. 2003. Comparação da morfologia foliar de espécies arbóreas de três estádios sucessionais distintos de floresta ombrófila densa (Floresta Atlântica) no Sul do Brasil. Revista Brasileira de Botânica 26: 61-72.

Brotto, M.L.; Santos, É.P. \& Baitello, J.B. 2009. Lauraceae no Morro dos Perdidos (Floresta Atlântica), Paraná, Brasil. Rodriguesia 60: 445-459.

Camerik, A.M. \& Werger, M.J.A. 1981. Leaf characteristics of the flora of the righ plateau of Itatiaia, Brasil. Biotropica 13: 39-48.

Carrasco, P.G. 2003. Produção de mudas de espécies florestais de restinga, com base em estudos florísticos e fitossociológicos, visando a recuperação de áreas degradadas, em Ilha Comprida/SP. Tese de Doutorado. Universidade Estadual Paulista Júlio de Mesquita Filho, Rio Claro/SP. 186p.

Carvalho, P.E.R. 2006. Espécies arbóreas brasileiras. Vol. 2. Embrapa Informação Tecnológica, Brasília; Embrapa Florestas, Colombo. 627p.

Clark, D.B. 1990. The role of disturbance in the regeneration of neotropical moist Forest. In: Bawa, K.S. \& Hadley, M. (eds.). Reproductive ecology of tropical forests plants. UNESCO/ The Parthenon Publishing, Paris. Pp. 291-315.

Clark, D.A. \& Clark, D.B. 1992. Life history diversity of canopy and emergent trees in a neotropical rain forest. Ecological Monographs 62: 315-344.

Denslow, J.S. 1996. Functional group diversity and responses to disturbance. In: Orians, G.H.; Dirzo, R.; Cushman, J.H. (eds.). Biodiversity and ecosystem processes in tropical forests. SpringerVerlag, Berlin. Pp. 127-151.

Durigan, G.; Nishikawa, D.L.L.; Rocha, E.; Silveira, E.R.; Pulitano, F.M.; Regalado, L.B. 1997. Manual de métodos de análise de solos. $2^{\mathrm{a}}$ ed. Embrapa - 
Empresa Brasileira de Pesquisa Agropecuária, Rio de Janeiro. 212p.

Durigan, G.; Nishikawa, D.L.L.; Rocha, E.; Silveira, E.R.; Pulitano, F.M.; Regalado, L.B.; Carvalhaes, M.A.; Paranaguá, P.A. \& Ramilu, V.E.L. 2002. Caracterização de dois estratos da vegetação em uma área de cerrado no município de Brotas, SPBrasil. Acta Botanica Brasilica 16: 251-262.

Embrapa - Empresa Brasileira de Pesquisa Agropecuária. 1997. Manual de métodos de análise de solos. 2.ed. Rio de Janeiro. 212p.

Fidalgo, A.O; Alcântara, R.P. \& Caldiron, G.T. 2009. Parâmetros de crescimento na avaliação de uma floresta implantada em uma restinga degradada pela mineração. Revista Brasileira de Biociência 4: 382-386.

Formento, S.; Schorn, L.A. \& Ramos, R.A.B. 2004. Dinâmica estrutural arbórea de uma Floresta Ombrófila Mista em Campo Belo do Sul, SC. Cerne 10: 196-212.

Franco, A.C.; Haag-Kerwer, A.; Herzog, B.; Grams, T.E.E.; Ball, E.; Mattos, E.A.; Scarano, F.R.; Barreto, S.M.B.; Garcia, M.A.; Mantovani, A. \& Lüttge, U. 1996. The effect of light levels on daily patterns of chlorophyll fluorescence and organic acid accumulation in the tropical CAM tree Clusia hilariana. Trees Structure and Function 10: 359-365.

Garwood, N.C. 1996. Functional morphology of tropical tree seedlings. In: Swaine, M.D (ed.). The ecology of tropical forest tree seedlings. Unesco, Paris. Pp. 59-129.

Gomes, F.H.; Vidal-Torrado, P.; Macias, F.; Gherardi, B. \& Perez, J.L.O. 2007. Solos sob vegetação de Restinga na Ilha do Cardoso (SP). I. Caracterização e classificação. Revista Brasileira de Ciência do Solo 31: 1563-1580.

Grime, J.P. 1977. Evidence for the existence of three primary strategies in plants and its relevance to ecological and evolutionary theory. The American Naturalist 982: 1169-1194.

Hoffmann, W.A. \& Franco, A.C. 2003. Comparative growth analysis of tropical Forest and savanna woody plants using phylogenetically independent contrasts. Journal of Ecology 91: 475-484.

Hunt, R. 1982. Plant growth curves. The functional approach to plant growth analysis. Edward Arnold, London. 248p.

Köppen, W.P. 1948. Climatología: con un estudio de los climas de la tierra. Fondo de Cultura Económica, México DC. 478p.

Kozlowski, T.T.; Kramer, P.J. \& Pallardy, S.G. 1991. The physiological ecology of woods plants. Academic Press, New York. 657p.

Kramer, P.J. \& Kozlowski, T.T. 1979. Physiology of wood plants. Academic Press, New York. 811p.
Lacerda, D.E.; Araujo, D. S. D \& Maciel, N. C. 1993. Dry coastal ecosystems of the tropical Brazilian coast. In: Van der Maarel, E. (ed.). Ecosystems of the world 2B: Dry Coastal Ecosystems Africa, America, Asia and Oceania. Elsevier, Amsterdam. Pp. 477-493.

Lima, R. A.F.; Oliveira, A.A.; Martini, A.M.Z.; Sampaio, D.; Souza, V.C. \& Rodrigues, R.R. 2011. Structure, diversity, and spatial patterns in a permanent plot of a high Restinga forest in Southeastern Brazil. Acta Botanica Brasilica 25: 633-645.

Magalhães, A.C.N. 1979. Análise quantitativa do crescimento. In: Ferri, M.G. Fisiologia vegetal. Universidade de São Paulo, São Paulo. Pp.331-350.

Magnago, L.F.S.; Martins, S.V.; Schaefer, C.E.G.R. \& Neri, A.V. 2010. Gradiente fitofisionômico-edáfico em formações florestais de Restinga no sudeste do Brasil. Acta Botanica Brasilica 24: 734-746.

Medina, E., Garcia,V. \& Cuevas, E. 1990. Sclerophylly and oligotrophic environments: relationships between leaf, structure, mineral nutrient content, and drought resistance in tropical rain forest of the upper Rio Negro region. Biotropica 22: 51-64.

Melo, M.R.F. \& Mantovani, W. 1994. Composição florística e estrutura fitossociológica da mata atlântica de encosta na Ilha do Cardoso (Cananéia, SP. Brasil). Boletim Instituto de Botânica 9:107-158.

Muller-Landau, H.C., Wright, S.J., Calderón, O., Hubbell, S.P. \& Foster, R. B. 2002. Assessing recruitment limitation: concepts, methods and case-studies from Tropical Forest. In: Levey, D.J.; Silva, W.R. \& Galetti, M. (eds). Seed dispersal and frugivory: ecology, evolution, and conservation. CABI Publishing, Wallingford. Pp. 35-53.

Pinheiro, M.H.O. \& Monteiro, R. 2006. Contribution of forest species to the floristic composition of a forested savanna in southeastern Brazil. Brazilian Archives of Biology and Technology 49: 763-774.

Pinheiro, M.H.O. \& Monteiro, R. 2009. Análise estrutural e considerações sobre a dinâmica sucessional de dois fragmentos florestais semideciduais do Jardim Botânico Municipal de Bauru, SP, Brasil. Acta Botanica Brasilica 23: 968-975.

Pinto, L.V.A.; Davide, A.C.; Botelho, S.A.; OliveiraFilho, A.T. \& Machado, E.L.M. 2005. Distribuição das espécies arbóreo-arbustivas ao longo do gradiente de umidade do solo de nascentes pontuais da bacia hidrográfica do ribeirão Santa Cruz, Lavras, MG. Cerne 11: 294-305.

Pires, L.A. 2006. Ecofisiologia de espécies ocorrentes em uma floresta de restinga. Tese de Doutorado. Universidade Estadual Paulista Julio de Mesquita Filho, Rio Claro. 251p.

Pires, L.A.; Cardoso, V.J.M.; Joly, C.A. \& Rodrigues, R.R. 2009. Germination of Ocotea pulchella (Nees) Mez (Lauraceae) seeds in laboratory and natural 
restinga environment conditions. Brazilian Journal of Biology 69: 935-942.

Pires, L.A. \& Cardoso, V.J.M. 2011. Effect of flooding on survival and initial growth of Ocotea pulchella (Nees) Mez. seedlings in semi-controlled light conditions. Acta Botanica Brasilica, 25: 435-444.

Pompa, J. \& Bongers, F. 1988. The effect of canopy gaps on growth and morphology of seedling of rain forest species. Oecologia 75: 625-632.

Poorter, L. 1999. Growth responses of 13 rain-forest tree species to a light gradient: the relative importance of morphological and physiological traits. Functional Ecology 13: 396-410.

Rodrigues, R. R.; Nave, A. 2004. Heterogeneidade Florística de Matas Ciliares. In: Rodrigues, R.R.; Leitão Filho, R.R. (orgs.). Matas Ciliares: Conservação e Recuperação. EDUSP/FAPESP, São Paulo. Pp: 45-72.

Salimon, C.I. \& Negrelle, R.B. 2001. Natural regeneration in a quarternary coastal plain in the Southern Brazilian Atlantic Rain Forest. Brazilian Archives of Biology and Technology 44: 155-163.

São Paulo (Estado). Secretaria do Meio Ambiente do Estado de São Paulo. Resolução SMA n.47, de 26 de novembro de 2003. Diário Oficial de São Paulo, 27 de novembro de 2003.

Shipley, B. 2002. Trade-offs between net assimilation rate and specific leaf area in determining relative growth rate: the relationship with daily irradiance. Functional Ecology 16: 682-689.
Shipley, B. 2006. Net assimilation rate, specific leaf area and leaf mass ratio: which is most closely correlated with relative growth? A meta-analysis. Functional Ecology 20: 565-574.

Silva, S.M. \& Britez, R.M. 2005. A vegetação da planície costeira. In: Marques, M.C.M. \& Britez, R.M. História natural e conservação da Ilha do Mel. Ed. UFPR, Curitiba. Pp. 49-84.

Smith, T. \& Huston, M. 1989. A theory of the spatial and temporal dynamics of plant communities. Vegetatio 83: 49-70.

Souza, R.P. \& Válio, I.F.M. 2003. Seedling growth of Brazilian tropical tree species differing in successional status. Revista Brasileira de Botânica 26: $35-47$.

Sugiyama, M. 2003. Estudos florísticos e fitossociológicos em comunidades vegetais de restinga da Ilha do Cardoso, Cananéia, SP. Tese de Doutorado. Universidade Federal de São Carlos, São Carlos. $134 \mathrm{p}$.

Yan, X.F. \& Cao, M. 2008. Effects of forest gap on the seedling growth of a canopy tree species Pometia tomentosa in tropical rainforest of Xishuangbanna. Ying Yong Sheg Tai Xue Bao 19: 238-44.

Zamith, L.R. \& Scarano, F.R. 2006. Restoration of a restinga sandy coastal plain in Brazil: survival and growth of planted woody species. Restoration Ecology, 14: 87-94.

Zar, J.H. 1996. Biostatistical analysis. Prentice-Hall International Editions, New Jersey. 662p. 